\title{
Correspondence
}

\author{
AIDS 2006, 20:000-000
}

\section{Concerns regarding a randomized trial of two postexposure prophylaxis regimens}

I would like to raise the following issues regarding your recent article by Gray et al. [1].

Perhaps as a result of the small sample size, exclusive and mixed breast-fed infants were combined into the same group. For some of the outcomes examined, keeping these groups separate is otherwise preferable.

Although the risk of mother-to-child transmission of HIV was twice as high in breast milk-exposed (BME) infants in multivariate analysis when the entire sample was included, the data for those receiving nevirapine, the option the authors clearly and with good reason suggest is preferred showed no statistically significant difference in the rates of mother-to-child transmission between BME infants and those not so exposed.

Among infants who received nevirapine, there was a $6.3 \%$ rate of transmission in the BME group at less than 10 days; the exclusively formula-fed group was $44 \%$ higher at 9.1\%. This was not tested for significance but sample sizes were small.

There has previously been speculation that early breast milk may be protective against transmission, which otherwise can occur at or near labor [2]. Perhaps a metaanalysis should be performed to examine the possibility in a larger sample size.

Rates of transmission in the BME group treated with 6 weeks of zidovudine increased rapidly: from $5.1 \%$ at less than 10 days to $14.5 \%$ at 6 weeks to $20.6 \%$ at 12 weeks. Because this is more rapid than has been seen in untreated infants [3], the possibility that longer-term postnatal dosing with zidovudine actually increases breast milk transmission rates cannot be ruled out. This possibility urgently needs to receive further research, given the
World Health Organization's recent decision to recommend the use of 4 weeks of zidovudine treatment to infants born to HIV-positive women who did not get prophylaxis during pregnancy [4].

The authors stated that there was 'no increased mortality in the formula-fed group' based on the lack of statistical significance of that group's $72 \%$ higher death rate $(3.8$ versus $2.2 \%$ ). This strikes me as a statement far beyond what the data support, given that the sample size was not adequate to measure mortality differences if they were indeed occurring.

Ted Greiner, PATH, $1800 \mathrm{~K}$ St NW, Suite 800, Washington, DC 20006, USA.

Received: 30 September 2005; accepted: 17 October 2005.

\section{References}

1. Gray GE, Urban $M$, Chersich MF, Bolton $C$, van Niekerk R, Violari A, et al., for the PEP Study Group. A randomized trial of two postexposure prophylaxis regimens to reduce motherto-child HIV-1 transmission in infants of untreated mothers. AIDS 2005; 19:1289-1297.

2. Coutsoudis A, Pillay K, Spooner E, Kuhn L, Coovadia HM. Influence of infant-feeding patterns on early mother-to-child transmission of HIV-1 in Durban, South Africa: a prospective cohort study. South African Vitamin A Study Group. Lancet 1999; 354:471-476.

3. Nduati R, John G, Mbori-Ngacha D, Richardson B, Overbaugh J, Mwatha $\mathrm{A}$, et al. Effect of breastfeeding and formula feeding on transmission of HIV-1: a randomized clinical trial. JAMA 2000; 283:1167-1174.

4. World Health Organization. Antiretroviral drugs and the prevention of mother-to-child transmission of HIV infection in resourcelimited settings. Recommendations for a Public Health Approach (2005 Revision). Available at: http://www.who.int/3by5/ PMTCTreport_June2005.pdf. Accessed: 3 November 2005. 


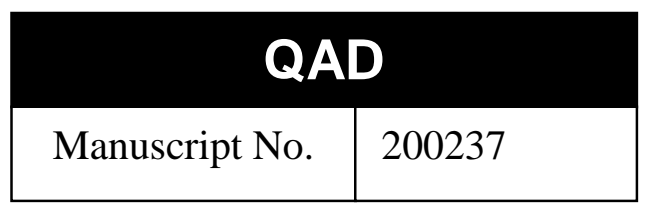
AIDS
Typeset by Thomson Digital for
Lippincott Williams \& Wilkins

Dear Author,

During the preparation of your manuscript for typesetting, some queries have arisen.

These are listed below. Please check your typeset proof carefully and mark any

corrections in the margin as neatly as possible or compile them as a separate list. This

form should then be returned with your marked proof/list of corrections to the Production Editor.

\section{QUERIES: to be answered by AUTHOR/EDITOR}

AUTHOR: The following queries have arisen during the editing of your manuscript. Please answer the queries by marking the requisite corrections at the appropriate positions in the text.

No Query. 